\title{
A DIFFERENTIAL-GEOMETRIC CRITERION FOR A SPACE CURVE TO BE CLOSED
}

\author{
HWANG CHENG-CHUNG
}

\begin{abstract}
In the present paper the author gives a necessary and sufficient condition to be satisfied by the curvature and torsion of a space curve in order to guarantee this curve to be closed (periodic).
\end{abstract}

In the lectures delivered by Professor Shiing-Shen Chern at the Institute of Mathematics of Academia Sinica when he visited Peking in June of 1978, he raised the following question:

What is the necessary and sufficient condition to be satisfied by the curvature and torsion, so that a space curve be a closed one?

The aim of the present paper is to give an answer.

Let $s$ denote the arc length of a space curve $(C)$ measured from a fixed point; and let $k(s)>0$ and $\sigma(s)$ be the curvature and torsion of $(C)$ respectively; let $\left[\vec{e}_{1}, \vec{e}_{2}, \vec{e}_{3}\right]$ denote an orthonormal basis in the Euclidean space $E^{3}$ of 3 dimensions, and we use

$$
\vec{x}=x_{1} \vec{e}_{1}+x_{2} \vec{e}_{2}+x_{3} \vec{e}_{3}
$$

to denote the position vector of a generic point. Let $\omega$ be the total length of $(C)$. When $(C)$ is a closed curve, the position vector $\vec{x}(s)$ must be a periodic function of period $\omega$. Here "period" is understood in the usual sense. Then from the formulas

$$
\text { Curvature } k(s)=\sqrt{\left\|\vec{x}^{\prime \prime}\right\|^{2}}, \quad \text { Torsion } \sigma(s)=k^{-2}\left(\vec{x}^{\prime}, \vec{x}^{\prime \prime}, \vec{x}^{\prime \prime \prime}\right)
$$

$\left(\vec{x}^{\prime}=d \vec{x} / d s\right.$, etc.) we find $k(s)$ and $\sigma(s)$ are also periodic of the same period. However, periodicity of $k(s)$ and $\sigma(s)$ are not sufficient to guarantee a space curve to be closed. For instance, the circular helix provides a counterexample.

In the following both $k(s)$ and $\sigma(s)$ are assumed to be continuous and periodic of period $\omega$, the vector function $\vec{x}(s)$ is therefore assumed of class $C^{3}$. Let $\vec{\xi}, \vec{\eta}, \vec{\zeta}$ denote the unit tangent, principal normal, and binormal vectors respectively at a generic point on the space curve $(C)$. Then we have the classical Frenet-Serret formulas

$$
d \vec{\xi} / d s=k \vec{\eta}, \quad d \vec{\eta} / d s=-k \vec{\xi}+\sigma \vec{\zeta}, \quad d \vec{\zeta} / d s=-\sigma \vec{\eta} .
$$

Writing $\vec{\xi}, \vec{\eta}, \vec{\zeta}$ in coordinate form as $\vec{\xi}=\sum_{i=1}^{3} \xi_{i} \vec{e}_{i}, \vec{\eta}=\sum_{i=1}^{3} \eta_{i} \vec{e}_{i}, \vec{\zeta}=\sum_{i=1}^{3} \zeta_{i} \vec{e}_{i}$ we find $\left(\xi_{1}, \eta_{1}, \zeta_{1}\right),\left(\xi_{2}, \eta_{2}, \zeta_{2}\right),\left(\xi_{3}, \eta_{3}, \zeta_{3}\right)$ are three independent solutions of the following system of differential equations:

$$
d \varphi_{1} / d s=k \varphi_{2}, \quad d \varphi_{2} / d s=-k \varphi_{1}+\sigma \varphi_{3}, d \varphi_{3} / d s=-\sigma \varphi_{2}
$$

Received by the editors August 13, 1980 and, in revised form, December 23, 1980.

1980 Mathematics Subject Classification. Primary 53C40. 
Obviously, (2) is a special case of the general equations

$$
d \varphi_{i} / d t=a_{i 1}(t) \varphi_{1}+a_{i 2}(t) \varphi_{2}+\cdots+a_{i n}(t) \varphi_{n} \quad(i=1,2, \ldots, n)
$$

where $a_{i j}(t)$ are assumed to be continuous and periodic of period $\omega$. Let the initial conditions be $\varphi_{i}(0)=\lambda_{i}$. For brevity, we write

$$
\varphi=\left(\begin{array}{c}
\varphi_{1} \\
\varphi_{2} \\
\vdots \\
\varphi_{n}
\end{array}\right), \quad \Phi(t)=\left(\begin{array}{cccc}
a_{11} & a_{12} & \ldots & a_{1 n} \\
a_{21} & a_{22} & \ldots & a_{2 n} \\
\ldots & \ldots & \ldots & \ldots \\
a_{n 1} & a_{n 2} & \ldots & a_{n n}
\end{array}\right)
$$

Then $\Phi(t)$ is a continuous periodic matrix function of period $\omega$. Let us make the conventions $\int_{0}^{t} \Phi(\tau) d \tau=\left(\int_{0}^{t} a_{i j}(\tau) d \tau\right)$, an $n \times n$ matrix, and

$$
\lambda=\left(\begin{array}{c}
\lambda_{1} \\
\lambda_{2} \\
\vdots \\
\lambda_{n}
\end{array}\right), \quad \varphi(t, \lambda)=\left(\begin{array}{c}
\varphi_{1}(t, \lambda) \\
\varphi_{2}(t, \lambda) \\
\vdots \\
\varphi_{n}(t, \lambda)
\end{array}\right)
$$

Then the equation (3) may be abbreviated to the form

$$
d \varphi / d t=\Phi(t) \varphi, \quad \varphi(0)=\lambda .
$$

The equations (4) are equivalent to the integral equation

$$
\varphi(t)=\lambda+\int_{0}^{t} \Phi(\tau) \varphi(\tau) d \tau
$$

for which both existence and uniqueness of solution are well known (for instance, see E. A. Coddington and N. Levinson, Theory of ordinary differential equations, McGraw-Hill, New York, 1955, p. 11), because continuity of $\Phi(t)$ has been assumed. Periodicity of $\varphi(t, \lambda)$ may be represented by

$$
\varphi(t+\omega, \lambda)=\varphi(t, \lambda) \quad(0<t<\omega) .
$$

As

$$
\begin{gathered}
\varphi(t, \lambda)=\lambda+\int_{0}^{t} \Phi(\tau) \varphi(\tau, \lambda) d \tau \\
\varphi(t+\omega, \lambda)=\lambda+\int_{0}^{t+\omega} \Phi(\tau) \varphi(\tau, \lambda) d \tau
\end{gathered}
$$

we have

$$
\begin{aligned}
\varphi(t+\omega, \lambda)-\varphi(t, \lambda)= & \int_{0}^{\omega} \Phi(\tau) \varphi(\tau, \lambda) d \tau \\
& +\int_{0}^{t} \Phi(\tau)[\varphi(\tau+\omega, \lambda)-\varphi(\tau, \lambda)] d \tau .
\end{aligned}
$$

If $\varphi(t, \lambda)$ is periodic in $t$, then by (6) we obtain

$$
\int_{0}^{\omega} \Phi(\tau) \varphi(\tau, \lambda) d \tau=0 .
$$

Conversely, assuming (7), we deduce $\varphi(t+\omega, \lambda)-\varphi(t, \lambda)$ is a solution of the integral equation $\psi(t)=\int_{0}^{t} \Phi(\tau) \psi(\tau) d \tau$. Then from uniqueness of solution we 
assert $\varphi(t+\omega, \lambda)-\varphi(t, \lambda)=0$. Thus we have proved the

LEMMA. The solution $\varphi(t, \lambda)$ of the equation

$$
d \varphi / d t=\Phi(t) \varphi, \quad \varphi(0)=\lambda
$$

$(\Phi(t+\omega) \equiv \Phi(t))$ is periodic of period $\omega$, if and only if

$$
\int_{0}^{\omega} \Phi(\tau) \varphi(\tau, \lambda) d \tau=0
$$

Now we review how the solution $\varphi(t, \lambda)$ is constructed. Recalling the well-known method of successive approximation due to E. Picard, let

$$
u_{0}=\lambda, \quad u_{n}(t)=\lambda+\int_{0}^{t} \Phi(\tau) u_{n-1}(\tau) d \tau \quad(n=1,2,3, \ldots)
$$

and pay attention to the facts:

$1^{\circ}$. The $u_{n}(t)$ are periodic vector functions of $t$.

$2^{\circ} .\left\{u_{n}(t)\right\}$ is uniformly convergent on the interval $[0, \omega]$.

$3^{\circ}$. Let $\lim _{n \rightarrow \infty} u_{n}(t)=\varphi(t, \lambda)$. Then $\varphi(t, \lambda)=\lambda+\int_{0}^{t} \Phi(\tau) \varphi(\tau, \lambda) d \tau$.

$4^{\circ}$. The $u_{n}(t)$ are linear in $\lambda$.

Let $(\mathcal{L} \Phi)(t) \equiv \mathscr{L}^{(1)} \Phi(t)=\int_{0}^{t} \Phi(\tau) d \tau$ and we define recursively, for $n>1$,

$$
\left(\mathcal{L}^{(n)} \Phi\right)(t)=\int_{0}^{t} \Phi(\tau)\left(\mathcal{L}^{(n-1)} \Phi\right)(\tau) d \tau
$$

Then

$$
\begin{aligned}
& u_{1}(t)=\{E+\mathcal{L} \Phi(t)\} \lambda \quad(E=\text { unit matrix }), \\
& u_{2}(t)=\left\{E+\mathcal{L} \Phi(t)+\mathcal{L}^{(2)} \Phi(t)\right\} \lambda, \\
& \cdots \\
& u_{n}(t)=\left\{E+\mathcal{L} \Phi(t)+\mathcal{L}^{(2)} \Phi(t)+\cdots+\mathcal{L}^{(n)} \Phi(t)\right\} \lambda .
\end{aligned}
$$

Let $n \rightarrow+\infty$. Then $\varphi(t, \lambda)=\left\{E+\mathcal{L} \Phi(t)+\cdots+\mathfrak{L}^{(n)} \Phi(t)+\cdots\right\} \lambda$, and hence

$$
\begin{aligned}
\int_{0}^{\omega} \Phi(t) \varphi(t, \lambda) d t & =\left\{\int_{0}^{\omega} \Phi(t) d t+\int_{0}^{\omega} \Phi(t) \mathcal{L} \Phi(t) d t+\cdots\right\} \\
& =\left\{\mathcal{L} \Phi(\omega)+\mathcal{L}^{(2)} \Phi(\omega)+\cdots+\mathcal{L}^{(n+1)} \Phi(\omega)+\cdots\right\} \lambda \\
& =M(\omega) \lambda,
\end{aligned}
$$

where the matrix $M(t)$ is defined by $M(t)=\mathcal{L} \Phi(t)+\mathfrak{L}^{(2)} \Phi(t)+\cdots+\mathcal{L}^{(n)} \Phi(t)$ $+\cdots$.

REMARK. In the special case that $\Phi$ commutes with $\mathcal{L} \Phi$ (for example, when $\Phi(t)$ is a constant matrix), the solution of the differential equation $d \varphi / d t=\Phi(t) \varphi$, $\varphi(0)=\lambda$ can be written explicitly as

$$
\varphi(t)=[\exp (\mathcal{E} \Phi)(t)] \lambda=\left[\exp \left(\int_{0}^{t} \Phi(\tau) d \tau\right)\right] \lambda
$$

[see, e.g., W. Hurewicz, Ordinary differential equations, M.I.T. Press, Cambridge, Mass., 1958, pp. 38-40]. In particular, the matrix $M(t)$ is given by

$$
M(t)=\left(\exp \int_{0}^{t} \Phi(\tau) d \tau\right)-E
$$


( $E=$ identity matrix).-This remark was added according to the suggestion of the referee, to whom the author wishes to express his deep gratitude.

Applying our lemma, we obtain

THEOREM I. The equations $d \varphi / d t=\Phi(t) \varphi$ possess a nonvanishing periodic solution of period $\omega$, if and only if $\operatorname{det}(M(\omega))=0$. In particular, in order that the equations $d \varphi / d t=\Phi(t) \varphi$ possess $n$ linearly independent periodic solutions of period $\omega$, the necessary and sufficient condition is that $M(\omega)$ be a zero matrix.

Now applying our Theorem I to the equations (2), then

$$
\begin{aligned}
& \Phi(s)=\left[\begin{array}{ccc}
0 & k(s) & 0 \\
-k(s) & 0 & \sigma(s) \\
0 & -\sigma(s) & 0
\end{array}\right), \\
& \mathcal{L} \Phi(s)=\left[\begin{array}{ccc}
0 & \int_{0}^{s} k(t) d t & 0 \\
-\int_{0}^{s} k(t) d t & 0 & \int_{0}^{s} \sigma(t) d t \\
0 & -\int_{0}^{s} \sigma(t) d t & 0
\end{array}\right] \\
& \mathcal{L}^{2} \Phi(\omega)=\left\{\begin{array}{cc}
-\int_{0}^{\omega} d s \int_{0}^{s} k(s) k(t) d t & 0 \\
0 & -\int_{0}^{\omega} d s \int_{0}^{s}[k(s) k(t)+\sigma(s) \sigma(t)] d t \\
\int_{0}^{\omega} d s \int_{0}^{s} \sigma(s) k(t) d t & 0
\end{array}\right. \\
& \int_{0}^{\omega} d s \int_{0}^{s} k(s) \sigma(t) d t \\
& -\int_{0}^{\omega} d s \int_{0}^{s} \sigma(s) \sigma(t) d t
\end{aligned}
$$

and so on. When and only when the matrix $M(\omega)$ is a zero matrix, there exist three mutually orthogonal unit vector functions $\vec{\xi}(s), \vec{\eta}(s), \vec{\zeta}(s)$ of period $\omega$, such that each set of functions $\left\{\xi_{i}(s), \eta_{i}(s), \zeta_{i}(s)\right\}(i=1,2,3)$ form a solution of the equation $d \varphi / d s=\Phi(s) \varphi(s)$ corresponding to the initial condition $\left(a_{i}, b_{i}, c_{i}\right)$. The vector function defining the space curve $(C)$ is

$$
\vec{x}(s)=\int_{0}^{s_{3}} \vec{\xi}(s) d s,
$$

where $\vec{\xi}(s)$ is given by

$$
\left[\begin{array}{l}
\xi_{i} \\
\eta_{i} \\
\zeta_{i}
\end{array}\right]=\left(\begin{array}{l}
a_{i} \\
b_{i} \\
c_{i}
\end{array}\right)+M(s)\left(\begin{array}{l}
a_{i} \\
b_{i} \\
c_{i}
\end{array}\right) \quad(i=1,2,3) .
$$

The curve $(C)$ is periodic with period $\omega$, if and only if $\int_{0}^{\omega} \vec{\xi}(s) d s=0$. Now let the initial conditions be $\xi_{i}(0)=a_{i}, \eta_{i}(0)=b_{i}, \zeta_{i}(0)=c_{i}(i=1,2,3)$, where $\left(a_{1}, a_{2}, a_{3}\right)$, $\left(b_{1}, b_{2}, b_{3}\right),\left(c_{1}, c_{2}, c_{3}\right)$ form an orthonormal frame. Then 


$$
\left[\begin{array}{c}
\xi_{i} \\
\eta_{i} \\
\zeta_{i}
\end{array}\right]=\left[\begin{array}{l}
a_{i} \\
b_{i} \\
c_{i}
\end{array}\right]+M(t)\left[\begin{array}{l}
a_{i} \\
b_{i} \\
c_{i}
\end{array}\right]
$$

i.e., $\xi_{i}=a_{i}+m_{11} a_{i}+m_{12} b_{i}+m_{13} c_{i}(i=1,2,3)$, so $\int_{0}^{\omega} \xi_{i}(s) d s=\omega a_{i}+a_{i} \int_{0}^{\omega} m_{11} d s$ $+b_{i} \int_{0}^{\omega} m_{12} d s+c_{i} \int_{0}^{\omega} m_{13} d s$. Since the determinant

$$
\left|\begin{array}{lll}
a_{1} & b_{1} & c_{1} \\
a_{2} & b_{2} & c_{2} \\
a_{3} & b_{3} & c_{3}
\end{array}\right| \neq 0
$$

the condition $\int_{0}^{\omega} \xi(s) d s=0$ is equivalent to $\omega+\int_{0}^{\omega} m_{11} d s=0=\int_{0}^{\omega} m_{12} d s=$ $\int_{0}^{\omega} m_{13} d s$. Thus we have proved the following

THEOREM II. Let $k(s)>0$ and $\sigma(s)$ be continuous periodic functions of period $\omega$ and let $(C)$ be a curve in $E^{3}$ with curvature $k$ and torsion $\sigma$. Then $(C)$ is periodic with period $\omega$, if and only if

(i) the matrix $M(\omega)=0$,

(ii) $\omega+\int_{0}^{\omega} m_{11} d s=0=\int_{0}^{\omega} m_{12} d s=\int_{0}^{\omega} m_{13} d s$, where

$$
\begin{gathered}
M(t)=\mathcal{L} \Phi(t)+\mathcal{L}^{(2)} \Phi(t)+\cdots+\mathcal{L}^{(n)} \Phi(t)+\cdots \\
\Phi(t)=\left[\begin{array}{ccc}
0 & k(t) & 0 \\
-k(t) & 0 & \sigma(t) \\
0 & -\sigma(t) & 0
\end{array}\right]
\end{gathered}
$$

and $m_{i j}(t)$ are the entries of the matrix $M(t)$.

Theorem II provides a decisive answer to the question asked by Professor Chern, which we have mentioned at the beginning of this paper. By means of this theorem we can verify the curve determined by

Curvature $k(s)=$ Const. $>0$ and Torsion $\sigma(s)=$ Const. is periodic of period $\omega$, if and only if $\sigma=0$ and $k=2 \pi / \omega$.

Remarks. 1. Although Theorem II is a decisive answer to Professor Chern's question, yet it is not quite satisfactory, for when this criterion is applied to a concrete problem, infinitely many integrations are involved. But it is doubtful whether this problem has a solution involving only a finite number of calculations at the present stage of mathematics.

2. It has been well known that for closed curves the total curvature and total torsion defined by $\int_{0}^{\omega} k(s) d s$ and $\int_{0}^{\omega} \sigma(s) d s$ are geometric invariants playing an important role in the theory of curves. It would be an interesting problem how to interpret geometrically the following invariants:

$$
\int_{0}^{\omega} d t \int_{0}^{t} k(t) k(s) d s, \quad \int_{0}^{\omega} d t \int_{0}^{t} k(s) \sigma(t) d s, \quad \int_{0}^{\omega} d t \int_{0}^{t} \sigma(t) \sigma(s) d s,
$$

etc.

Department of Mathematics, Nanking University, Nanking, China 